\title{
Lithium depletion and the rotational history of exoplanet host stars
}

\author{
J. Bouvier
}

\author{
Laboratoire d'Astrophysique, Observatoire de Grenoble, Université J. Fourier, CNRS, UMR 5571, BP 53, \\ 38041 Grenoble Cedex 9, France \\ e-mail: jbouvier@obs.ujf-grenoble.fr
}

Received 11 July 2008 / Accepted 26 August 2008

\section{ABSTRACT}

\begin{abstract}
Context. It has been reported that exoplanet host stars are lithium depleted compared to solar-type stars without detected massive planets.

Aims. We investigate whether enhanced lithium depletion in exoplanet host stars may result from their rotational history. Methods. We have developed rotational evolution models for slow and fast solar-type rotators from the pre-main sequence (PMS) to the age of the Sun and compare them to the distribution of rotational periods observed for solar-type stars between 1 Myr and 5 Gyr. Results. We show that slow rotators develop a high degree of differential rotation between the radiative core and the convective envelope, while fast rotators evolve with little core-envelope decoupling. We suggest that strong differential rotation at the base of the convective envelope is responsible for enhanced lithium depletion in slow rotators.

Conclusions. We conclude that lithium-depleted exoplanet host stars were slow rotators on the zero-age main sequence (ZAMS) and argue that slow rotation results from a long lasting star-disk interaction during the PMS. Altogether, this suggests that long-lived disks ( $\geq 5 \mathrm{Myr}$ ) may be a necessary condition for massive planet formation/migration.
\end{abstract}

Key words. planetary systems: formation - stars: rotation - stars: abundances - stars: pre-main sequence accretion, accretion disks - hydrodynamics

\section{Introduction}

As the discovery rate of exoplanets has steadily increased over the years, now reaching nearly 300 detections, interest has grown in the properties of their host stars (Santos et al. 2003; Kashyap et al. 2008). Israelian et al. (2004) report that solar-type stars with massive planets are more lithium-depleted than their siblings without detected massive planets. This result has recently been confirmed by Gonzalez (2008). Lithium over-depletion in massive exoplanet hosts appears to be a generic feature over a restricted $T_{\text {eff }}$ range from 5800 to $5950 \mathrm{~K}$, independent of the planet's orbital properties. Metallicity effects and/or the early accretion of planetesimals have been ruled out as the origin of lithium over-depletion in exoplanet hosts (Castro et al. 2008). Instead, it has been suggested that enhanced lithium burning could stem from the tidal effect of the giant planet on the host star, as it induces rotationally-driven mixing in the stellar interior (Israelian et al. 2004). However, the mass of giant exoplanets is much lower than the mass of the convective envelope of solar-type stars $\left(\geq 0.02 M_{\odot}\right)$. In addition, enhanced lithium depletion is seen for stars with giant planets that span a wide range of semi-major axes, up to about 2 AU. Whether the tidal interaction between a distant massive planet and the host star can be strong enough to induce rotationally-driven mixing remains to be investigated.

Rotationally-driven mixing may instead be related to the rotational history of the star (Takeda et al. 2007; Gonzalez 2008). We revisit here the link between lithium depletion and the rotational history of solar-type stars, and discuss its relationship with massive planet formation. In Sect. 2, we present models for the rotational evolution of solar-type stars from the early PMS to the age of the Sun. We use these models to fit the most recent measurements of rotational periods obtained for solartype stars in star forming regions and young open clusters. We discuss the properties of 2 extreme models: one reproduces the evolution of fast rotators over time, and the other the evolution of slow rotators. We find that slow rotators develop a high degree of differential rotation between the inner radiative zone and the outer convective envelope, while fast rotators evolve with little core-envelope decoupling. From these results, we investigate in Sect. 3 the evolution of lithium abundances in slow and fast rotators. We suggest that the large velocity shear at the core/envelope interface in slow rotators leads to more efficient Li-depletion. In Sect. 4, we relate the Li-depletion pattern of massive exoplanet host stars to their rotational history. We argue that both are dictated by their initial angular momentum and the lifetime of their protoplanetary disk. We conclude that long-lived disk may be a necessary condition for massive planet formation/migration around young solar-type stars.

\section{The rotational history of solar-type stars}

\subsection{Rotational evolution models}

The rotational evolution of solar-mass stars depends on a number of physical processes acting over the star's lifetime. The models dicussed here were originally developed by Bouvier et al. (1997) and Allain (1998), and we apply them to the most recent observational constraints available today. The reader is referred to the original papers for a complete description of the models, but we only briefly recall the main assumptions and physical ingredients. The rotational evolution of low-mass stars goes through 3 main stages: PMS evolution, ZAMS approach, and MS relaxation. During the early PMS evolution, the young star is 
magnetically coupled to its accretion disk. As long as this interaction lasts, the star is prevented from spinning up as it contracts and evolves at constant angular velocity. This "disk-locking" assumption is supported by both observational evidence (Rebull et al. 2006; Cieza \& Baliber 2007) and MHD models of the star-disk interaction (e.g. Matt \& Pudritz 2005; Romanova et al. 2007). The disk lifetime is a free parameter of the model, and it dictates the early rotational evolution of the star. Then, as the disk dissipates after a few Myr, the star spins up as it contracts and becomes more centrally-condensed (due to the development of an inner radiative zone) on its approach to the ZAMS $^{1}$. Depending on the initial velocity and the disk lifetime, a wide range of rotation rates can be obtained on the ZAMS (cf. Bouvier et al. 1997). The longer the disk lifetime and the lower the initial velocity, the slower the rotation rate on the ZAMS. In contrast, high initial velocities and/or short disk lifetimes lead to fast rotation on the ZAMS. Finally, as the stellar internal structure stabilizes at an age of about 40 Myr for a solar-mass star, the braking by a magnetized wind becomes the dominant process and effectively spins the star down on the early MS. As the braking rate scales with surface velocity (see Kawaler 1988; Bouvier et al. 1997), fast rotators are spun down more efficiently than slow ones. As a result, and in spite of a large dispersion of rotation rates on the ZAMS, solar-type stars converge towards uniformly slow rotation by the age of the Sun. Indeed, after a few Gyr, the surface rotational velocity of solar-type stars has lost all memory of the past rotational history.

Internal differential rotation is an important additional parameter of this model. The response of the stellar interior to angular momentum loss at the stellar surface affects the evolution of the surface rotation rate. We follow Allain (1998) in considering a radiative core and a convective envelope that are each in rigid rotation, but whose rotation rates may differ. We therefore introduce a coupling timescale between the inner radiative zone and the outer convective envelope, $\tau_{\mathrm{c}}$, which measures the rate of angular momentum transfer between the core and the envelope (MacGregor \& Brenner 1991). A short coupling timescale corresponds to an efficient core-envelope angular momentum transport and, as a consequence, little internal differential rotation. In contrast, a long coupling timescale will lead to the developement of a large rotational velocity gradient between the core and the envelope. This model parameter is thus expected to be essential for evaluating the amount of rotationally-induced mixing and associated lithium depletion during the evolution of solartype stars.

\subsection{Model fitting: slow and fast rotators}

The models are constrained by the rotation rates of solar-type stars at various ages. In the last years, rotational periods have been measured for hundreds of low-mass stars in molecular clouds (1-5 Myr) and young open clusters (40-600 Myr), thus providing a tight observational sampling of the rotational evolution of low-mass stars. Table 1 lists the 10th and 75th percentiles of the derived rotational period distributions for stars with a mass between 0.8 and $1.1 M_{\odot}$ and belonging to various stellar clusters with an age between $1 \mathrm{Myr}$ and $625 \mathrm{Myr}$. These values were converted to angular velocity $(\omega=2 \pi / P)$, normalized to the Sun's angular velocity, and are plotted as a function of time in Fig. 1.

We aim here at reproducing the lower and upper envelopes of the observed rotational distributions, in order to contrast the

\footnotetext{
${ }^{1}$ We used the $1 M_{\odot}$ model from Baraffe et al. (1998) to follow the evolution of the star's internal structure.
}

Table 1. Observational datasets: 10th and 75th percentiles of the rotational period distribution for $0.8-1.1 M_{\odot}$ stars.

\begin{tabular}{llllll}
\hline \hline Name & $\begin{array}{l}\text { Age } \\
(\mathrm{Myr})\end{array}$ & $\begin{array}{l}P_{10 \%} \\
(\mathrm{~d})\end{array}$ & $\begin{array}{l}P_{75 \%} \\
(\mathrm{~d})\end{array}$ & $N_{\text {star }}$ & Ref. \\
\hline ONC & 1 & 1.4 & 8.9 & 35 & 1 \\
NGC 2264 & 2 & 1.0 & 4.5 & 16 & 2,3 \\
NGC 2362 & 5 & 1.5 & 9.8 & 40 & 4 \\
NGC 2547 & 40 & 0.35 & 6.8 & 15 & 5 \\
Pleiades & 120 & 0.44 & 6.1 & 11 & 6 \\
M 50 & 130 & 0.59 & 8.7 & 74 & 7 \\
M 34 & 200 & 0.89 & 7.2 & 22 & 8 \\
M 37 & 550 & 3.6 & 7.3 & 145 & 9 \\
Hyades & 625 & 5.4 & 9.1 & 15 & 10 \\
\hline
\end{tabular}

(1) Herbst et al. (2002); (2) Lamm et al. (2005); (3) Makidon et al. (2004); (4) Irwin et al. (2008b); (5) Irwin et al. (2008a); (6) Compilation (see references in Irwin et al. 2008a); (7) Irwin (priv. comm.); (8) Irwin et al. (2006); (9) Hartman et al. (2008); (10) Radick et al. (1987, 1995).

evolution of slow and fast rotators and relate it to lithium depletion. A model for fast rotators is compared to observations in Fig. 1. Starting from an initial period of $1.2 \mathrm{~d}$, the star remains coupled to its disk for $5 \mathrm{Myr}$, then spins up to a velocity of about $160 \mathrm{~km} \mathrm{~s}^{-1}$ on the ZAMS, and is eventually spun down by a magnetized wind on the MS to the Sun's velocity. This model fits reasonably well the PMS spin up and the rapid MS spin down observed for fast rotators between 5 and $500 \mathrm{Myr}$. To reach this agreement, the core-envelope coupling timescale has to be short, $\tau_{\mathrm{c}} \sim 10 \mathrm{Myr}$. A longer coupling timescale would lead to envelope spin down before the star reaches the ZAMS, and a slower spin down rate on the early MS, both of which would conflict with observations. The tight coupling between the core and the envelope implies that little differential rotation develops in fast rotators, with the rotation of the core barely exceeding that of the envelope on the early MS (see Fig. 1).

Figure 1 also shows a model for slow rotators. The initial period is $10 \mathrm{~d}$, and the star-disk interaction lasts for $5 \mathrm{Myr}$ in the early PMS. As the star approaches the ZAMS, both the outer convective envelope and the inner radiative core spin up. Once on the ZAMS, the outer envelope is quickly braked, while the core remains in rapid rotation. This behavior results from an assumed weak coupling between the core and the envelope, with $\tau_{\mathrm{c}} \sim 100 \mathrm{Myr}$. On the early MS, the rapidly-rotating core transfers angular momentum back to the envelope, which explains the nearly constant surface velocity over several $100 \mathrm{Myr}$ in spite of magnetic braking. A long coupling timescale between the core and the envelope is thus required to account for the observed rotational evolution of slow rotators. A shorter coupling timescale would lead to higher ZAMS velocities and a sharper spin down on the early MS, which both would conflict with observations. A long $\tau_{\mathrm{c}}$ in slow rotators implies inefficient transport of internal angular momentum and results in a large velocity gradient at the core-envelope boundary (see Fig. 1).

The main difference between fast and slow rotator models is thus twofold: the initial angular momentum and the level of core-envelope decoupling. The initial angular momentum of fast rotators is 10 times greater than that of slow ones. Fast rotators lose more angular momentum over the course of their evolution than slow rotators, as they both converge towards the Sun's velocity at 4.65 Gyr. The lower panels in Fig. 1 show the magnitude of the rotational shear at the base of the convective envelope for slow and fast rotators, and the amount of angular momentum transported from the core to the envelope. Differential rotation 

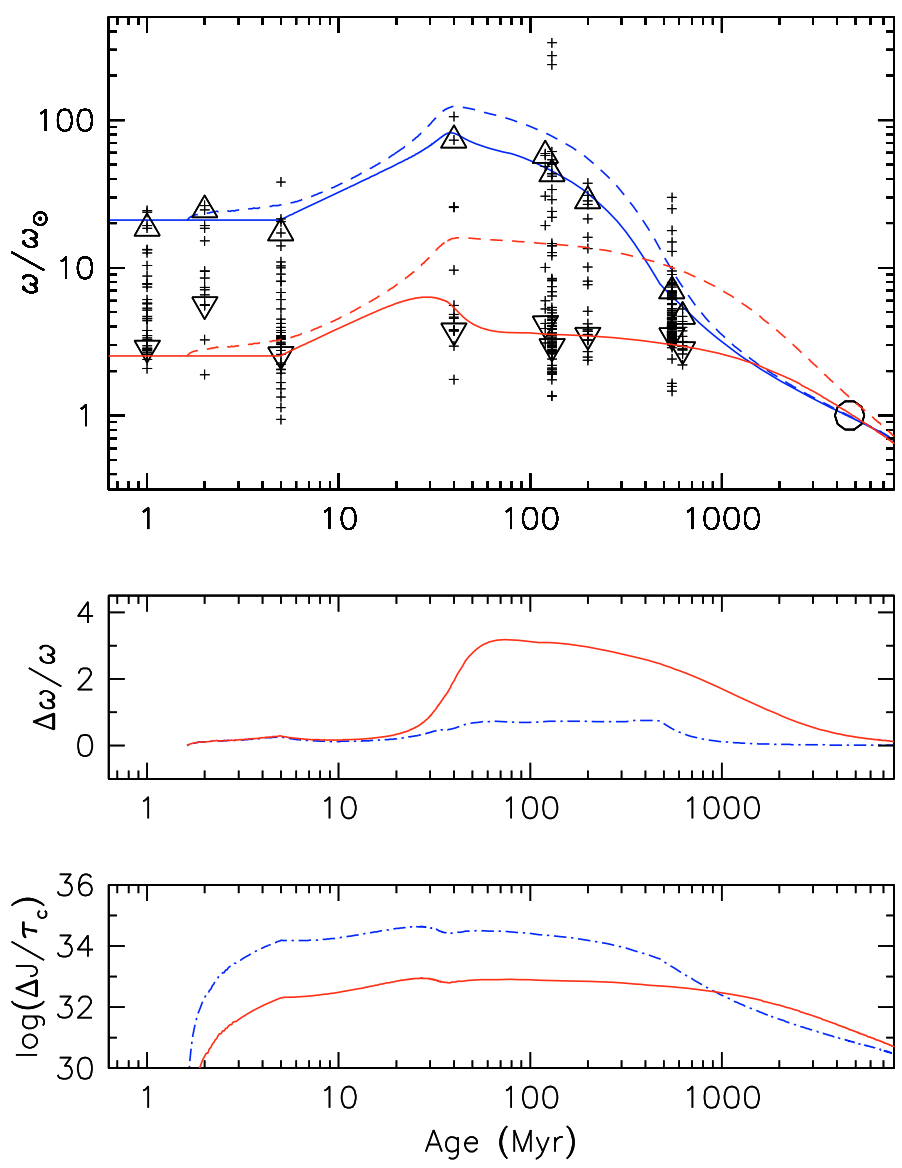

Fig. 1. Rotational models for slow and fast rotators. Data: the 10th and 75 th percentiles of the observed rotational period distributions were converted to angular velocity and are plotted as direct and inverted triangles as a function of time. Individual measurements of rotational periods converted to angular velocities are also shown in order to illustrate the statistical significance of the various samples (see Table 1). Models: the modeled evolution of surface rotation for slow and fast rotators is shown by the solid lines. For both models, the rotation of the radiative core is shown by the dashed lines. With a core-envelope coupling timescale of only $10 \mathrm{Myr}$, little differential rotation develops in fast rotators. In contrast, the $100 \mathrm{Myr}$ core-envelope coupling timescale in slow rotators leads to the development of a large velocity gradient at the base of the convective zone. A disk lifetime of $5 \mathrm{Myr}$ is assumed for both models. Lower panels: the velocity shear at the base of the convective zone $\left(\omega_{\text {rad }}-\omega_{\text {conv }}\right) / \omega_{\text {conv }}$ and the angular momentum transport rate $\Delta J / \tau_{\mathrm{c}}\left(\mathrm{g} \mathrm{cm}^{2} \mathrm{~s}^{-2}\right)$ from the core to the envelope are shown for slow (solid line) and fast (dotted-dashed line) rotators.

develops in slow rotators at the ZAMS and remains strong during early MS evolution until the core and the envelope eventually recouple after a few Gyr. Clearly, slow rotators exhibit a much larger rotational shear at the base of their convective envelope than do fast rotators during most of their evolution. Conversely, angular momentum transport from the core to the envelope is much more efficient in fast rotators than in slow ones, which leads to very little differential rotation indeed. Rotational shear and angular momentum transport are both directly related to the core-envelope coupling timescale and are independent of the assumed disk lifetimes. Obviously, the different amounts of internal differential rotation between slow and fast rotators is expected to affect their lithium depletion pattern.

\section{Lithium depletion in slow and fast rotators}

Hydrodynamical instabilities acting on various timescales are responsible for the so-called rotationally-driven mixing that increases the efficiency of lithium burning in stellar interiors (Talon 2008). The main sources of rotational mixing are shearinduced turbulence and large-scale meridional circulation, both of which are linked to internal differential rotation (Zahn 2007). These processes transport angular momentum and chemical species, though not necessarily at the same rate. Due to the complex physics involved, it is by no means staightforward to predict from first principles which instability dominates in solar-type stars over the course of their evolution. Some guidance is provided by observations. A key result in this respect is the observed relationship between $\mathrm{Li}$ abundances and rotation in young stars. Soderblom et al. (1993) report that fast solar-type stars in the Pleiades exhibit higher Li abundances than slow rotators. Taken at face value, this result indicates that Li depletion already takes place during the PMS, and is more pronounced in slow rotators than in fast ones. Hence, rotationally-induced mixing appears more efficient in slow rotators.

Going back to the models described above, this result suggests that rotational mixing is primarily driven by the rotational shear at the base of the convective envelope: slow rotators are observed to be more Li-depleted on the ZAMS than fast rotators and the models presented here indicate that they experience stronger core-envelope decoupling. In contrast, fast rotators lose more angular momentum at the stellar surface and also transfer angular momentum more efficiently from the core to the envelope. Yet, they seem to preserve most of their initial lithium during the PMS. Efficient angular momentum transport is apparently not associated with a significant mixing of chemical species in these stars. Mixing-free processes for angular momentum transport have to be sought. Candidates include magnetic field (Eggenberger et al. 2005) and internal gravity waves (Talon et al. 2002). Whether magnetic fields, whether of fossil origin or generated by a dynamo at the tachocline, can efficiently couple the core to the envelope is still under debate (Brun \& Zahn 2006). And while gravity waves seem capable of removing angular momentum from the core, the efficiency of this process and the amount of associated chemical mixing still remain to be fully explored (Charbonnel \& Talon 2005).

Comparison between rotational models and lithium measurements on the ZAMS thus suggests that shear-induced turbulence associated to core-envelope decoupling is the dominant source of rotationally-driven lithium depletion in solar-type stars. Our finding that core-envelope decoupling is stronger in slower rotators is at odds with most previous theoretical studies. In Pinsonneault et al. (1989) models, for instance, the rotational shear at the base of the convective envelope scales with surface velocity. Hence, fast rotators are predicted to be more heavily Li-depleted than slow ones. We show here instead that, to be able to account for the observed rotational evolution of solartype stars from the PMS to the age of the Sun, one has to assume that the velocity gradient at the base of the convective envelope is larger in slow rotators than in fast ones (see also Bouvier 1997). As a result, slow rotators on the ZAMS are expected to be more efficiently Li-depleted than fast rotators, as observed.

\section{Lithium depletion and planet formation}

We discussed above how different rotational histories may affect the lithium depletion pattern of solar-type stars and thus lead to a dispersion of lithium abundances in these stars at a given age and mass. The scenario outlined above suggests that enhanced 
lithium depletion is associated to low surface rotation on the ZAMS. Then, that mature solar-type stars with massive exoplanets are Li-depleted compared to similar stars with no planet detection seems to indicate that massive exoplanet hosts had slow rotation rates on the ZAMS.

Why were massive exoplanet host stars slow rotators on the ZAMS? Two main parameters govern the rotation rate on the ZAMS: the initial velocity and the disk lifetime. For any given disk lifetime, the lower the initial velocity, the lower the velocity on the ZAMS. Conversely, for any given initial velocity, the longer the disk lifetime, the lower the velocity on the ZAMS. This is because the magnetic star-disk interaction during the PMS is far more efficient than solar-type winds in extracting angular momentum from the star (Bouvier 2007; Matt \& Pudritz 2007). Disk lifetimes varying from star to star in the range $1-10 \mathrm{Myr}$ are required to account for the distribution of rotational velocities on the ZAMS (Bouvier et al. 1997). Statistically, however, the slowest rotators on the ZAMS are expected to be the stars that initially had low rotation rates and the longest-lived disks.

Long-lived disks may thus appear as a necessary condition for massive planet formation and/or migration on a timescale $\geq 5$ Myr. Long lasting disks may indeed be the common origin for slow rotation on the ZAMS, Li-depletion, and massive planet formation. Interestingly enough, the Sun is a massive exoplanet host. Even though the solar system gaseous planets are located farther away from the Sun than massive exoplanets are from their host stars, the Sun is strongly Li-deficient. According to the scenario outlined above, the Sun would thus have been a slow rotator on the ZAMS. More generally, another intriguing result is the apparent bimodality of $\mathrm{Li}$ abundances in mature solar-type stars (e.g. Pasquini et al. 1994, 2008). In the framework of the model developed here, it is interesting to note that the rotational distribution of young stars also appears bimodal (Choi \& Herbst 1996). Whether the bimodal distributions of rotation and lithium could be related through the rotational history of solar-type stars remains to be addressed.

\section{Conclusions}

Based on what we currently know of the rotational properties of young stars, of the lithium depletion process in stellar interiors, and of the angular momentum evolution of solar-type stars, it seems likely that the lithium-depleted content of massive exoplanet host stars is a sequel to their specific rotational history. This history is predominantly dictated by star-disk interaction during the PMS. Using simple rotational models, it is suggested here that the long disk lifetimes of initially slow rotators lead to the development of a large velocity shear at the base of the convective zone. The velocity gradient in turn triggers hydrodynamical instabilities responsible for enhanced lithium burning on PMS and MS evolution timescales. Rotationally-driven lithium depletion in exoplanet host stars can thus be at least qualitatively accounted for by assuming PMS disk lifetimes of 5-10 Myr. Such long-lived disks may be a necessary condition for planet formation and/or migration around young solar-type stars, at least for the class of giant exoplanets detected so far (Ida \& Lin 2008).

Admittedly, the scenario outlined here is based on rotational models that do not incorporate a detailed physical description of the processes at work. The conclusion therefore remains qualitative and has to be ascertained by the development of more sophisticated angular momentum evolution models. Most important, the transport processes that lead to a strong core-envelope coupling in fast rotators and a weak one in slow rotators still have to be identified. While this goal is well beyond the scope of the present study, we hope that the simplified models presented here may provide useful guidelines for further refinements.

Acknowledgements. It is a pleasure to acknowledge discussions of transport processes in stellar interiors with C. Charbonnel, S. Mathis, A. Palacios, S. TurckChieze, and J.-P. Zahn, during the 30th meeting of the French Astronomical Society in Paris. I thank I. Baraffe for providing the $1 M_{\odot}$ model, and J. Irwin for providing M50's rotational data before publication.

\section{References}

Allain, S. 1998, A\&A, 333, 629

Baraffe, I., Chabrier, G., Allard, F., \& Hauschildt, P. H. 1998, A\&A, 337, 403 Bouvier, J. 1997, Mem. Soc. Astron. Ital., 68, 881

Bouvier, J. 2007, IAU Symp., 243, 231

Bouvier, J., Forestini, M., \& Allain, S. 1997, A\&A, 326, 1023

Brun, A. S., \& Zahn, J.-P. 2006, A\&A, 457, 665

Castro, M., Vauclair, S., Richard, O., \& Santos, N. C. 2008, Mem. Soc. Astron. Ital., 79, 679

Charbonnel, C., \& Talon, S. 2008, [arXiv: 0805 . 4697]

Choi, P. I., \& Herbst, W. 1996, AJ, 111, 283

Cieza, L., \& Baliber, N. 2007, ApJ, 671, 605

Eggenberger, P., Maeder, A., \& Meynet, G. 2005, A\&A, 440, L9

Gonzalez, G. 2008, MNRAS, 386, 928

Hartman, J. D., Gaudi, B. S., Pinsonneault, M. H., et al. 2008, [arXiv: 0803.1488]

Herbst, W., Bailer-Jones, C. A. L., Mundt, R., Meisenheimer, K., \& Wackermann, R. 2002, A\&A, 396, 513

Ida, S., \& Lin, D. N. C. 2008, ApJ, 673, 487

Irwin, J., Aigrain, S., Hodgkin, S., et al. 2006, MNRAS, 370, 954 Irwin, J., Hodgkin, S., Aigrain, S., et al. 2008a, MNRAS, 383, 1588 Irwin, J., Hodgkin, S., Aigrain, S., et al. 2008b, MNRAS, 384, 675

Israelian, G., Santos, N. C., Mayor, M., \& Rebolo, R. 2004, A\&A, 414, 601

Kashyap, V. L., Drake, J. J., \& Saar, S. H. 2008, [arXiv : 0807 . 1308]

Kawaler, S. D. 1988, ApJ, 333, 236

Lamm, M. H., Mundt, R., Bailer-Jones, C. A. L., \& Herbst, W. 2005, A\&A, 430, 1005

MacGregor, K. B., \& Brenner, M. 1991, ApJ, 376, 204

Makidon, R. B., Rebull, L. M., Strom, S. E., Adams, M. T., \& Patten, B. M. 2004, AJ, 127, 2228

Matt, S., \& Pudritz, R. E. 2005, ApJ, 632, L135

Matt, S., \& Pudritz, R. E. 2007, IAU Symp., 243, 299

Pasquini, L., Liu, Q., \& Pallavicini, R. 1994, A\&A, 287, 191

Pasquini, L., Biazzo, K., Bonifacio, P., Randich, S., \& Bedin, L. 2008, [arXiv:0807.0092]

Pinsonneault, M. H., Kawaler, S. D., Sofia, S., \& Demarque, P. 1989, ApJ, 338, 424

Radick, R. R., Thompson, D. T., Lockwood, G. W., Duncan, D. K., \& Baggett, W. E. 1987, ApJ, 321, 459

Radick, R. R., Lockwood, G. W., Skiff, B. A., \& Thompson, D. T. 1995, ApJ, 452, 332

Rebull, L. M., Stauffer, J. R., Megeath, S. T., Hora, J. L., \& Hartmann, L. 2006, ApJ, 646, 297

Romanova, M. M., Long, M., Kulkarni, A. K., et al. 2007, IAU Symp., 243, 277

Santos, N. C., Israelian, G., Mayor, M., Rebolo, R., \& Udry, S. 2003, A\&A, 398, 363

Soderblom, D. R., Jones, B. F., Balachandran, S., et al. 1993, AJ, 106, 1059

Takeda, Y., Kawanomoto, S., Honda, S., Ando, H., \& Sakurai, T. 2007, A\&A, 468,663

Talon, S. 2008, Mem. Soc. Astron. Ital., 79, 569

Talon, S., Kumar, P., \& Zahn, J.-P. 2002, ApJ, 574, L175

Zahn, J.-P. 2007, EAS Publ. Ser., 26, 49 